\title{
Cap sur le premier MOOC FOFLE en Afrique francophone pour se (re)mettre à flot
}

\author{
Ghedhahem, Zeineb
}

Ecole Polytechnique de Tunisie, zeineb_3112@yahoo.fr

\begin{abstract}
Resumen
Tecnologías ofrecen oportunidades para hacer la enseñanza y el aprendizaje sea más efectivo y adecuado, y nadie puede negar los beneficios potenciales de la revolución digital en la enseñanza de idiomas. Una nueva forma de lenguaje de enseñanza a distancia atrae a más y más alumnos : MOOCs (Massive Open Course on-line) - en francés, CIOMS (Curso en Línea Abierta y sólido). Sin nivel de estrés de la educación o la asistencia, el MOOC puede ofrecer el aprendizaje en línea y la distancia con un seguimiento educativo específico y recursos educativos (vídeos, documentos, tareas...) accesible en todo momento. El MOOC FOFLE (Formación Abierta en francés como lengua extranjera) es un proyecto piloto, internacional e interdisciplinario dedicado a aprender francés. Se puso a prueba en Túnez antes de ser desplegados en otros países de habla francesa con el mismo problema. Nuestro objetivo en esta reunión es presentar las principales características de este dispositivo y la primera evaluación realizada.
\end{abstract}

Palabras clave : MOOC ; la educación ; aprendizaje ; la enseñanza de idiomas ; interdisciplinario

\begin{abstract}
Résumé
Les technologies offrent des possibilités de rendre l'enseignement et l'apprentissage plus efficaces et plus appropriés et nul ne peut nier les potentiels bénéfices de la révolution numérique dans l'enseignement des langues. Une nouvelle forme d'enseignement des langues à distance attire de plus en plus d'apprenants : les MOOCs (Massive Open Online Course) en français, CLOMs (Cours en Ligne Ouverts et Massifs). Sans contrainte de niveau d'études, ni d'assiduité, le MOOC permet d'offrir un apprentissage en ligne et à distance avec un suivi pédagogique spécifique et des ressources pédagogiques (vidéos, documents, devoirs...) accessibles à tout moment. Le MOOC FOFLE (Formation Ouverte en Français Langue Etrangère) est un projet pilote, international et interdisciplinaire, dédié à l'apprentissage en ligne du français. Il a été expérimenté en Tunisie: avant d'être déployé à destination des autres pays francophones présentant la même problématique. Notre objectif au cours de cette rencontre est de présenter les caractéristiques principales de ce dispositif ainsi que le premier bilan effectué.
\end{abstract}

Mots-clés : MOOC ; enseignement ; apprentissage en ligne; enseignement des langues ; interdisciplinaire.

\begin{abstract}
Technologies offer opportunities to make teaching and learning more effective and appropriate and no one can deny the potential benefits of the digital revolution in language teaching. A new form of teaching distance language attracts more and more learners : MOOCs (Massive Open Online Course) in French, CIOMS (Online Course Open and Solid). Without stress level of education or attendance, the MOOC can offer online learning and distance with a specific educational monitoring and educational resources (videos, documents, homework ...) accessible at all times. The MOOC FOFLE (Open Training in French as a Foreign Language) is a pilot, international and interdisciplinary project dedicated to learning French. It was piloted in Tunisia before being deployed to other francophone countries with the same problem. Our goal during this meeting is to present the main features of this device and the first assessment done.
\end{abstract}

Keywords : $M O O C$; education ; learning ; language teaching ; interdisciplinary. 
Alors que le français est la langue d'enseignement et d'apprentissage dans le cycle d'enseignement supérieur tunisien, différentes études de terrain ont montré que l'usage de cette langue est loin d'être systématique et que les références à la langue arabe (arabe dialectal) sont fréquentes notamment dans les filières scientifiques et techniques. L'on note par ailleurs que la langue française est de moins en moins bien maîtrisée par les étudiants tunisiens, toutes disciplines confondues. Or, dans l'optique d'une amélioration qualitative de l'université tunisienne, la maîtrise des langues étrangères et notamment de la langue française est un point essentiel. De plus, ces lacunes relatives aux compétences linguistiques, et particulièrement à la langue française, représentent un double handicap pour les étudiants : dans la poursuite de leur cursus universitaire et par voie de conséquence, leur potentiel d'employabilité immédiat et futur.

En 2014, un projet visant à remédier humblement à cette situation, a vu le jour en Tunisie, en proposant aux élèves terminant leur enseignement secondaire ou aux étudiants entrant à l'université de suivre des sessions d'apprentissage du français langue étrangère sous la forme d'un MOOC FOFLE à dimension résolument collaborative. Cette expérience pilote a vu le jour grâce au partenariat entre l'Université Virtuelle de Tunis, l'Université de Sfax et le Consortium Claroline Connect. Dans cette phase d'expérimentation, il était question de tester ce dispositif en vue de le déployer par la suite à destination d'autres pays francophones africains où la situation est quasi-similaire.

Entamé le 13 octobre 2014, Le MOOC FOFLE, projet pilote et première expérience en Tunisie et en Afrique, a été achevée au mois de mars 2015. Pour cette première session, nombreux étaient les étudiants qui ont bénéficié d'une formation en ligne en vue d'améliorer leur niveau en français, aussi bien à l'écrit qu'à l'oral, utilisant pour cela du contenu riche et varié (texte, quizz, vidéos,...) et se faisant accompagner également par des tuteurs de terrain et des tuteurs référents. Bien évidemment, l'objectif était de renforcer leurs compétences générales pour, entre autres, être des mieux outillés à la sortie de l’université. J’y ai personnellement contribué en tant que tutrice- référent.

Au cours de cette rencontre, je vais vous rendre compte de cette expérience pilote en vous présentant le dispositif mis en place ainsi que le bilan final.

Pour ce faire, mon travail s'articulera autour de trois volets. Dans le premier, je procéderai à la présentation du dispositif, à savoir le MOOC. Les deux autres volets seront consacrés, respectivement, aux différents acteurs impliqués ainsi qu'aux différentes modalités d'évaluation. Je conclurai mon intervention par le bilan de cette première expérience du MOOC en Tunisie.

\section{Le MOOC}

\section{Pourquoi un MOOC de FLE?}

Depuis 2008, les MOOCs ne cessent de se multiplier, un vrai raz de marée. Cela concerne aussi bien les matières scientifiques que les langues, notamment la langue anglaise. Dès lors, pourquoi ne pas en concevoir un en français langue étrangère ? D'autant plus que nous sommes à la recherche d'une solution pour résoudre le problème de la détérioration de la maitrise de la langue française en Tunisie et dans d'autres pays francophones.

Le premier MOOC de FLE conçu par Alix Creuzé ${ }^{1}$ et Jérôme Rambert² ${ }^{2}$ a été lancé le 15 janvier 2014. Cette première initiative " Travailler en français » a été animée de janvier à mars 2014 par une équipe de professeurs de FLE répartis en Allemagne, Espagne, Italie et au Royaume-Uni. Il s'est adressé à des apprenants de français du monde entier, à tous ceux qui souhaitaient un jour travailler en français ou avec des francophones. Le thème du MOOC «Travailler en français » était exclusivement lié au monde du travail. Or le MOOC FOFLE était destiné à des étudiants ainsi qu'à des élèves inscrits au programme "Education numérique ». Ce projet expérimental est censé venir en aide aux étudiants en difficulté et leur permettre de mieux réussir au cours de leur cursus universitaire. La nouveauté réside dans le fait que le MOOC est pensé de manière à proposer une approche originale fondée sur la mise à disposition d'un MOOC en association avec un modèle d'accompagnement tenant compte principalement des besoins des apprenants. L'autre innovation dans ce dispositif concerne les tuteurs de terrain. En effet, ces derniers sont des étudiants en master FLE

\footnotetext{
${ }^{1}$ Professeur conceptrice et coordinatrice des dispositifs à distance et de l'innovation pédagogique à l’Institut français d'Espagne.

${ }^{2}$ Professeur de FLE, coordinateur pédagogique et formateur de formateurs en Tice à l’Institut français de Milan.
} 
ayant exprimé le désir de se former dans le tutorat. Tuteurs de terrain et apprenants suivent des formations en ligne de concert. Dès lors, ce projet se présente comme un véritable catalyseur d’innovation et un élément d'intégration pour tous.

Le contenu des différents modules du MOOC FOFLE a été conçu pour des apprenants du niveau A2 du CECR désirant atteindre le niveau B1. Les objectifs généraux étaient, d’une part, l’amélioration de la compréhension et de la production aussi bien orales qu'écrites afin de permettre aux apprenants inscrits d'achever leurs cursus avec succès et d'autre part, le renforcement de leurs compétences générales pour, entre autres, être des mieux outillés à la sortie de l'université. Au cours de cette phase d'expérimentation, le public ciblé était les étudiants néo bacheliers entrant à l'université tunisienne. L'objectif étant de toucher un maximum de ceux-ci, en particulier à Tunis et à Sfax en attendant d'étendre cela aux étudiants des autres niveaux et d'autres institutions aussi bien tunisiennes qu'africaines.

\section{Les objectifs}

- Linguistiques : compétences linguistiques niveau A2/B1 - priorité à la « Compétence de compréhension ».

- Méthodologiques : initiation à la méthodologie universitaire.

- Culturels et interculturels : acculturation universitaire et approche interculturelle des documents et des thématiques.

- Période d'expérimentation : octobre 2014 à mars 2015.

De type connectiviste, le MOOC FOFLE était composé de 6 séquences de formation, chaque apprenant devait y consacrer 6 heures de travail par séquence, soit un total de 36 heures réparties sur 12 semaines. Les activités proposées étaient aussi bien individuelles que collaboratives. La conception de ces séquences était uniforme : les activités proposées gravitaient autour des 4 compétences de base à savoir la compréhension ainsi que la production de l'oral et de l'écrit, certains points de langue étaient intégrés selon les objectifs discursifs. Une activité collaborative d’intégration clôt chaque séquence ainsi qu’une évaluation.

\section{Les thèmes}

Les concepteurs ont jugé bon de privilégier les thèmes de culture générale, vu que les apprenants venaient de divers horizons. Ainsi les thèmes retenus étaient les suivants :

1. Internet et numérique,

2. Environnement et progrès,

3. Découvertes et avenir,

4. Sciences et recherche,

5. Arts et société,

6. Médias et échanges internationaux.

Une séquence d'initiation à l'utilisation de la plateforme Claroline Connect a été programmée au début de la formation afin de familiariser les apprenants avec ce nouvel espace d'apprentissage d'autant plus que la majorité d'entre eux découvrent pour la première fois l'enseignement en ligne.

\section{Les activités}

Pour aider l'apprenant à prendre conscience des mécanismes du français parlé, un travail d'exploration leur a été proposé au début de chaque séquence par des tâches d’écoute du français tel qu’il est parlé (extraits vidéos) suivi de quelques activités de compréhension/ production pour améliorer la compétence langagière. Ainsi les activités proposées étaient diverses : 
- des activités auto-correctives de compréhension de la langue écrite et orale accompagnées de quelques activités relatives à des points de langue ciblés,

- des activités de production orale avec tuteur sur rendez-vous hangout et/ou des activités de production écrite individuelle et collaborative,

- des activités d'auto-évaluation,

- des tâches collectives, en petits groupes ou en binômes, d'expression orale ou écrite.

\section{Les acteurs}

Le tutorat

L'accompagnement a été confié à des tuteurs francophones, même si certains n’avaient aucune expérience en ce qui concerne l'enseignement à distance. Ils ont néanmoins bénéficié d'une formation accélérée au cours du premier semestre 2014.

Les tuteurs relevaient de deux catégories distinctes : des tuteurs de terrain encadrés par des tuteurs référents.

- Les tuteurs de terrain : 18 étudiants de Master2 en FLE issus des universités françaises n'ayant pas nécessairement bénéficié d'une expérience de tutorat en ligne, ces tuteurs sont formés au cours du MOOC à travers une formation en ligne, assurée par les tuteurs référents. Au terme de cette formation, un certificat attestant des connaissances et compétences acquises en tant que tuteurs de formation FLE en ligne leur a été délivré.

Chaque tuteur avait pris en charge 20 apprenants. Leurs missions consistaient d'une part en l'accompagnement des apprenants au quotidien à travers les outils de communication dont disposait la plateforme: forums, blogs, Hangout, etc...et d'autre part à l'évaluation des productions écrite et orales des apprenants.

- Les tuteurs référents étaient des enseignants de français tunisiens ayant une bonne connaissance de l'université tunisienne ainsi qu'une expérience de l'usage des TICEs et du tutorat en ligne, idéalement dans le cadre de formations en français langue étrangère (FLE) ou en français sur objectifs spécifiques (FOS). Leurs missions consistaient en l'accompagnement des tuteurs de terrain et la supervision du déroulement du Mooc. Chaque tuteur référent avait pour mission d'encadrer 2 à 3 tuteurs de terrain Ils pouvaient en outre intervenir en tant que tuteurs de terrain.

\section{L'environnement numérique d'apprentissage}

En complément à la plateforme Claroline Connect, une communauté MOOC FOFLE sur Google+ (le réseau social de Google) a été créée permettant de la sorte à l'ensemble des participants au MOOC d'échanger réactions, idées, questions, invitations à discuter, etc. de manière plus souple et moins formelle.

Pour assurer les séances synchrones, le choix des administrateurs techniques s'est fixé sur le service de webconférences de Google (Hangout).

\section{Les apprenants}

600 personnes se sont inscrites au MOOC, nous considérons ce chiffre plutôt satisfaisant dans le sens où c'est une première en Tunisie. Le nombre aurait pu augmenter si nous avions permis aux retardataires de s'inscrire. Malheureusement, au-delà d'une certaine date, les inscriptions étaient fermées. Nous avons pu identifier des profils différents (néo bacheliers, étudiants inscrits en 2éme et 3éme année de licence ainsi que des étudiants du Master, des fonctionnaires....). Les apprenants actifs, enclins au partage et au travail collaboratif étaient très minoritaires. La majorité était plutôt du genre passif. Sur les 600 inscrits, seuls 50 apprenants sont allés jusqu'au bout et ont achevé le parcours et ont pu obtenir les badges après les épreuves d'évaluation. 
Les apprenants, à l'unanimité, se sont trouvés dans un environnement peu familier et face à un challenge de taille. En effet, ils devaient affronter 3 difficultés :

- Suivre une formation en langue française,

- $\quad$ Utiliser de nouveaux outils techniques,

- s’accommoder d'une pédagogie où priment la collaboration et l'auto-apprentissage.

Le travail collaboratif n’a pas répondu à nos attentes, le bon vieux réflexe, «s'en remettre à l'enseignant », a la peau dure. Même si nous encourageons les apprenants à prendre l'initiative, à devenir plus autonomes, il leur est encore difficile de sauter le pas. Les difficultés majeures auxquelles étaient confrontés les apprenants étaient d’ordre psychoaffectif, comme la constitution des équipes, des binômes....ils avaient beaucoup de mal à choisir des partenaires car ils se considéraient comme des étrangers, le sentiment d'appartenance à une communauté était quasi nul. Ils ont du mal à tisser des liens entre eux à travers la toile. A l'avenir nous devrions tenir davantage compte de certains aspects comme le sentiment de sécurité, la confiance, la solidarité, le soutien, l'existence d’une communauté, la complicité. Tout un travail reste à faire concernant l'environnement de l'enseignement à distance ainsi que les tâches proposées. La culture de l'apprentissage en réseau n'est pas encore instaurée en Tunisie et beaucoup de travail reste à faire en ce sens. C'est en multipliant type de pratique que tuteurs et apprenants finiront par acquérir suffisamment d'expérience pour pallier ce manque.

\section{L'évaluation}

L'évaluation de l'apprenant constitue une phase fondamentale de la formation MOOC puisqu'elle permet de traduire le degré de validité des connaissances acquises. Deux méthodes différentes d'évaluation ont été choisies par les concepteurs : l'évaluation automatisée et l'évaluation par les pairs. L'évaluation se fait tout au long de la formation, elle s'effectue pendant le MOOC pour évaluer l'état d'avancement et de positionnement de l'apprenant et à sa fin pour délivrer des badges à l'apprenant une fois qu'il a atteint le seuil de réussite.

L'évaluation automatisée recouvre un large éventail de techniques. Elle se base sur des outils assez rudimentaires comme les questionnaires à choix multiples ou les textes à trous aussi bien que sur des techniques plus avancées comme les programmes de test de codes ou d'évaluation automatisée de copies.

Souvent on pense que l'évaluation automatisée, ce sont seulement des quiz. Mais cela va bien au-delà ; cela recouvre un large éventail de techniques : les QCM, les textes à trous, les applications numériques, mais aussi des techniques plus avancées comme des programmes et des algorithmes développés et qui évaluent automatiquement les productions. Mais nous n'en parlerons pas dans cette intervention, car il y a déjà beaucoup de choses à dire.

L'évaluation par les pairs consiste à faire évaluer une production d'un participant par un ou plusieurs de ses pairs. Elle permet d'une part le passage à l'échelle de l'évaluation lorsqu'il n'est pas possible de se baser sur une technique d'évaluation automatisée; c'est la fonction diagnostique de l'évaluation. Mais elle a également une fonction pédagogique, évaluer une copie nécessite un travail de réflexion conséquent; c’est la fonction formative de l’évaluation.

Il existe de nombreuses recherches sur l'évaluation par les pairs. En particulier Sadler et Good ont montré que l'évaluation quantitative par les pairs et l'autoévaluation sont proches de l'évaluation donnée par les enseignants.

En outre l'évaluation par les pairs permet d'apprendre, en effet, cette forme d'évaluation met en œuvre un apprentissage mutuel et une prise de recul par rapport à ses propres productions. L'apprentissage mutuel est en étroite relation avec l'évaluation par les pairs. Une fois son travail évalué par ses pairs, l'apprenant revoit ses textes initiaux en tenant compte des avis formulés précédemment. 


\section{Le bilan du projet}

\section{Les retours des participants}

Dans l'ensemble, les apprenants étaient satisfaits de la formation, le seul bémol était l'absence de séance de présentiel. Ils en avaient besoin pour se retrouver dans un environnement plus familier et surtout voir « de près » les différents acteurs. Le fait de ne pas pouvoir s'exprimer en langue arabe, chose qu'ils font systématiquement dans les formations classiques, a rebuté plus d'un.

\section{Du coté tuteurs}

- proposer des parcours aux niveaux de difficulté distincts, correspondant aux différents niveaux d'engagement des participants. Tous ces apprenants ont nécessairement des attentes, des connaissances de base ou encore des manières d'apprendre très différentes. Or, actuellement, il n’y a qu'un seul et unique parcours dans un MOOC, qui ne conviendra pas forcément à tous. Outre le fait que le processus d'apprentissage soit ainsi loin d'être optimal pour chacun des apprenants, les frustrations et découragements qui peuvent alors en découler ont pour conséquence une autre problématique majeure pour les MOOCs : le dropout (abandon).

- Oser les échanges synchrones ouverts où les participants parlent d'eux.

- Le tutorat dans les MOOC devrait être assuré par des enseignants ayant de grandes compétences dans les TICEs, ce qui leur permettrait de personnaliser les contenus en fonction des différents besoins.

\section{Conclusion}

Le problème majeur est sans conteste le besoin d'organiser l'accompagnement des apprenants. Le schéma envisagé est certainement idéal et peut fonctionner, à condition de disposer d'un nombre suffisant de tuteurs, ce qui n'est pas certain, du moins en comptant sur le seul bénévolat.

Il conviendrait par ailleurs de mieux préparer les apprenants potentiels à aborder le MOOC en les amenant à suivre des formations qui les mettent au niveau requis pour suivre une formation en ligne, singulièrement en termes de maîtrise des outils informatiques. Cette réorganisation de la formation implique entre autres une réorganisation des contenus, voire le développement de contenus nouveaux.

Les MOOC représentent un défi de taille pour l'enseignement supérieur en Tunisie. Dans le sillage des grandes universités américaines, la plupart des établissements cherchent à se positionner dans ce domaine. Malgré son apparent succès, le système est loin d'être parfait et beaucoup de progrès reste à faire. L'absence de diplômes reconnus est l'un des principaux inconvénients de ces cours en ligne. Ils offrent néanmoins une formidable opportunité pour approfondir ses connaissances dans une multitude de domaines et ouvrent sans doute la voie à la pédagogie virtuelle de demain. Le MOOC est un « objet pédagogique » comme un autre. Ce qui m'intéresse c'est son côté social, ouvert et déclencheur de prise de conscience. Les Massive Open Online Courses ne sont qu'une étape, mais cela permet de se poser des questions sur la place du numérique dans la pédagogie. Il y aura sans doute d’autres innovations demain.

\section{Références bibliographiques}

KIM, Sun-Mi et VERRIER, Christian (2009). Le plaisir d'apprendre en ligne à l’Université. Bruxelles : de Boeck.

Miled, Mohamed ; BECHA, Jacqueline et BENREJEB, Bourguiba (2008). Référentiel de Compétences «Langue » pour les 3 années L1, L2 et L3, PREFSUP, Tunis.

Msalmi, Mohamed (2013). L'enseignement du FOS dans le contexte universitaire tunisien après la réforme LMD. Enjeux institutionnels et démarches didactiques, Thèse de Doctorat soutenue à l’Université Virtuelle de Tunis.

SAHNOUn, Mokthar (2006). Le français aujourd'hui, nº 154, Armand Colin.

SAID, Mosbah (2008). Tunisie, Langue-culture, interculturalité ou expression de sa propre lecture dans la langue de l'autre : faut-il privilégier un vecteur? Cas de l'enseignement du français dans le contexte tunisien. Publications université d'Aristote Thessalonique. Dans : Actes du colloque Communiquer dans les langues cultures, Thessalonique, Grèce, pp. 475-486. 